\title{
Southern range extensions for twelve heterobranch sea slugs (Gastropoda: Heterobranchia) on the eastern coast of Australia
}

Matt J. Nimbs ${ }^{1,2^{*}}$, Meryl Larkin ${ }^{1,2}$, Tom R. Davis ${ }^{1,2}$, David Harasti ${ }^{3}$, Richard C. Willan ${ }^{4}$ and Stephen D. A. Smith ${ }^{1,2}$

\begin{abstract}
Port Stephens, on the central New South Wales coast, provides ideal oceanographic and benthic conditions for the settlement and growth of larvae of tropical species delivered from the north by the East Australian Current. The popularity of the bay for recreational and scientific diving has facilitated extensive documentation of the biota over several decades, confirming its high biodiversity. Of the 313 species of heterobranch sea slugs recorded from Port Stephens to date, 30 are not known to occur further south. Our observations increase the number of taxa with a southern distribution limit at Port Stephens by 12 species and add to a growing list of marine taxa that are progressively extending their southern range, potentially as a result of climate change.
\end{abstract}

Keywords: Port Stephens, Opisthobranchs, Nudibranchs, Climate change, Citizen science

\section{Introduction}

Heterobranch sea slugs (hereafter simply sea slugs) are predominantly tropical marine animals that, on the eastern Australian coast, exhibit a latitudinal gradient with rapid attenuation of species diversity from more than 1,000 species in the northern Great Barrier Reef (GBR) to approximately 500 in central New South Wales (NSW) (Rudman \& Willan, 1998). The southward flow of the East Australian Current (EAC) brings warm water from tropical latitudes to the Tasman Sea (Booth et al., 2007; Malcolm et al., 2010) and with it planktonic larvae (Booth et al., 2007; Malcolm et al., 2010). With increasing latitude, the current moves offshore and generates eddies (mostly in summer) that deliver an intermittent supply of these tropical larvae to the central and southern NSW coast (Burn, 2006).

South-eastern Australia is a recognised climate change 'hot-spot' (Hobday \& Lough, 2011) where strengthening

\footnotetext{
* Correspondence: matt.nimbs@gmail.com

${ }^{1}$ National Marine Science Centre, Southern Cross University, P.O. Box 4321, Coffs Harbour, NSW 2450, Australia

${ }^{2}$ Marine Ecology Research Centre, Southern Cross University, Lismore, NSW 2456, Australia

Full list of author information is available at the end of the article
}

of the EAC and increasing water temperature may facilitate the arrival and establishment of novel species that may alter species interactions (Underwood \& Chapman, 2007). In this area, southward shifts in distribution are anticipated for many marine organisms (Przeslawski et al., 2008), with range extensions already documented for some species of fishes (Figueira \& Booth, 2010; Harasti, 2015), scleractinian corals (Baird et al., 2012), sea slugs (Nimbs et al., 2015), and host anemones and their complement of commensal crustaceans (Scott et al., 2015). New species records will come about through greater intensity of observations (sea slugs are inherently rare in time and space - Marshall \& Willan, 1999) and as a result of range shifts due to warming conditions. Those changes resulting from warming seas have important implications for marine conservation management particularly for species with a very restricted range (O'Hara 1995).

Comprehensive data on the occurrence of a range of marine organisms tend to be confined to locations near major population centres due primarily to the proximity of research facilities (Smith 2005, 2008a, b; Burn, 2006). For this reason, species lists for the central NSW coast, especially adjacent to Sydney, are comprehensive, 


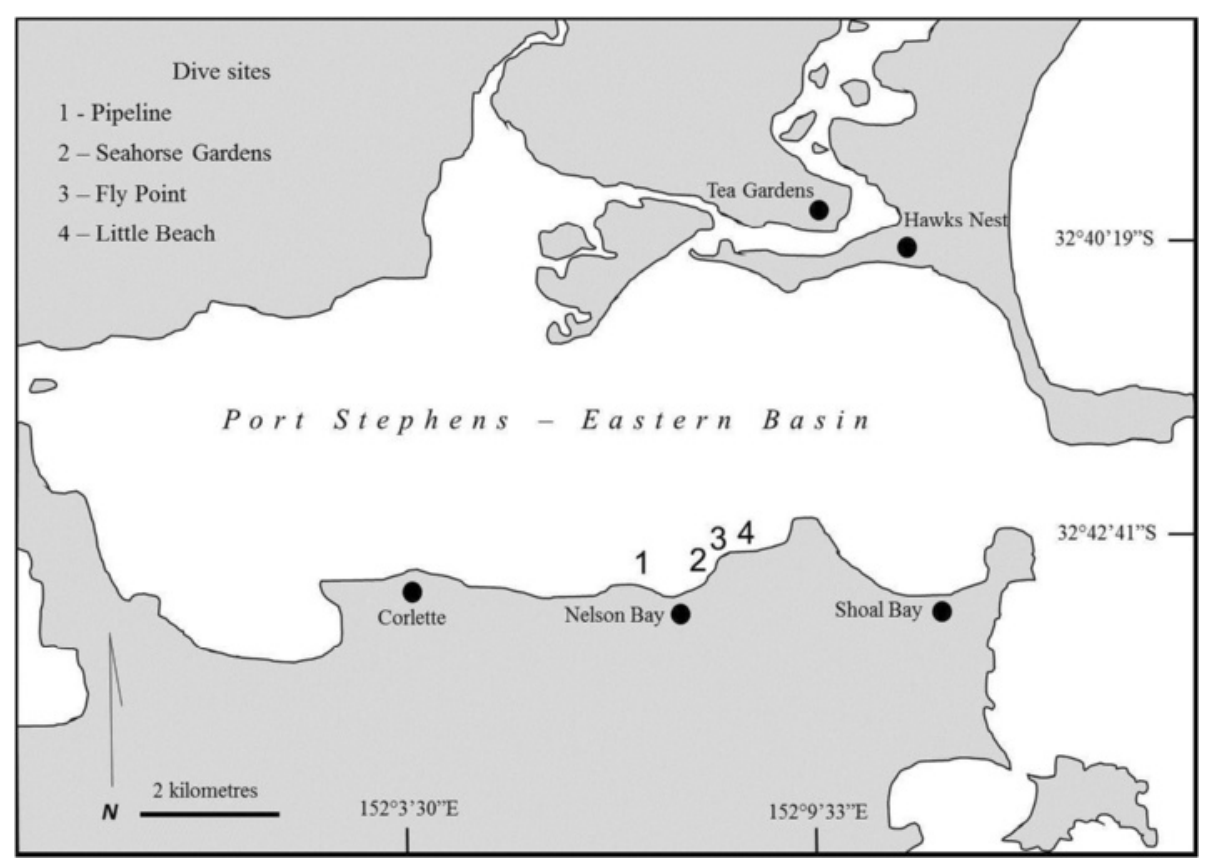

Fig. 1 Map of Port Stephens eastern basin with dive sites indicated. Shading represents land

particularly for fishes (Gladstone, 2007; Morton \& Gladstone, 2011; Harasti et al., 2015). The occurrence and distribution of sea slugs is also well known, however to record range extensions reliably, it is imperative that observations of any taxa not previously recorded in an area are thoroughly documented.

The Port Stephens-Great Lakes Marine Park encompasses 98,000 ha of coastal and shelf waters between Cape Hawke and Birubi Beach, incorporating the extensive Port Stephens embayment (Fig. 1). Within the marine park, varying oceanographic conditions, sea bed topography and sessile assemblages contribute to a high species diversity that is a mixture of tropical, subtropical and temperate flora and fauna (DECCW, 2010, Smith et al., 2010). Being close to Newcastle, the second largest city in NSW, and with easy access for SCUBA divers and watercraft, the biotic and abiotic aspects of Port

Table 1 Selected records of Philinopsis orientalis from Australian waters

\begin{tabular}{llll}
\hline Location & Record coordinates & Year & Reference \\
\hline Lizard & $14^{\circ} 40^{\prime} 30^{\prime \prime} \mathrm{S}$ & 1982 & Pers. obs. (R. C. Willan) \\
Island, QLD & $145^{\circ} 26^{\prime} 26^{\prime \prime} \mathrm{E}$ & & \\
Coates & $17^{\circ} 10^{\prime} 53^{\prime \prime} \mathrm{S}$ & 1997 & Pers. obs. (R. C. Willan) \\
Reef, QLD & $146^{\circ} 22^{\prime} 10^{\prime \prime} \mathrm{E}$ & & \\
Rottnest & $31^{\circ} 59^{\prime} 19^{\prime \prime} \mathrm{S}$ & $1990-2000$ & (Wells \& Bryce \\
Island, WA & $115^{\circ} 30^{\prime} 10^{\prime \prime} \mathrm{E}$ & & 2000, p. 31-32) \\
Nelson & $32^{\circ} 43^{\prime} 13^{\prime \prime} \mathrm{S}$ & 2015 & This paper \\
Bay, NSW & $152^{\circ} 08^{\prime} 39^{\prime \prime} \mathrm{E}$ & & \\
\hline
\end{tabular}

Stephens have been well studied (e.g. Harasti et al., 2014; Poulos et al., 2015; Davis et al., 2015).

Many sea slug species of tropical origin are regularly observed in Port Stephens where conditions are conducive for the settlement of veliger larvae that may have travelled considerable distance on the EAC. In this paper, observations of 12 sea slug species are recorded at significant distances (at least $300 \mathrm{~km}$ ) south of their previously reported southern limit. Coastal lagoons and estuaries provide sheltered locations for successful settlement and growth of more tropically adapted marine organisms (Willan et al., 1979) and accessible, safe diving conditions. These factors, combined with the increasing popularity of diving-based citizen science activities in the region (e.g. Smith \& Edgar, 2014 and the current Sea Slug Census program) increase the likelihood that additional species will not only occur in the region, but also that they have a reasonable probability of being found.

\section{Materials and methods}

Port Stephens is a large, drowned river valley fed by two major, eastward-flowing rivers, the Karuah and the Myall, and comprises two basins that exhibit differences in substrate and hydrodynamics. The marine-influenced eastern basin is characterised by a complex of channels and shoals formed by the influence of strong tidal flows (Vila-Concejo et al., 2007) with a diverse range of marine habitats (Davis et al., 2015). A narrow entrance and elevated shoreline to the south-east provide considerable 


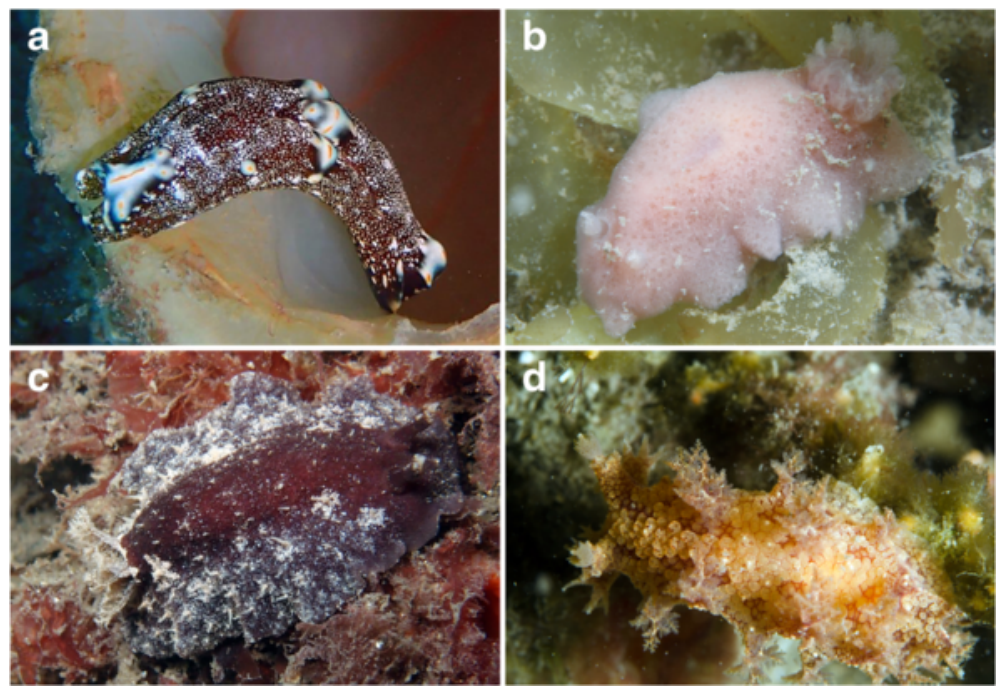

Fig. 2 a. Philinopsis orientalis. Fly Point, 14 February, 2015. b. Jorunna ramicola. Pipeline, 30 November, 2013. c. Thordisa tahala. Little Beach, 15 January, 2015. d. Marionia pustulosa. Seahorse Gardens, 7 March, 2015. Photos: a, Matt Doyle; b, c, Tom Davis; d, Kristine O'Keefe

protection from the effects of strong southerly winds and large ocean swells. The tidal range in the sheltered port is approximately $1.4 \mathrm{~m}$ (Creese \& Wales, 2009) and the average depth is $14 \mathrm{~m}$ (Poulos et al., 2015).

Organic input from rivers and tidal flow contribute considerable volumes of food to support suspensionfeeding organisms such as sponges, ascidians, octocorals and hydroids (Smith 2008a, b; Smith et al., 2010). These organisms, in turn, provide food and habitat for a diverse assemblage of sea slugs. Several popular shore-dive sites, noted for their diverse invertebrate life and opportunities for macro-photography, are located on the southern shoreline of the eastern basin, centered around

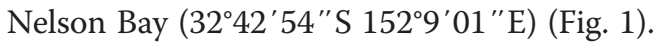

The 'Pipeline' supports a mixed habitat comprising rocky reef with large macrophytes and sandy sediments with seagrasses, sponges and octocorals (Harasti \& Gladstone, 2013). 'Seahorse Gardens' is $900 \mathrm{~m}$ east of the 'Pipeline' and supports octocoral colonies situated in sandy substrate with sponges located in deeper areas (Harasti et al., 2014). 'Fly Point' lies to the east of 'Seahorse Gardens' within a sanctuary (no take) zone that

Table 2 Records of Jorunna ramicola from Australian waters

\begin{tabular}{llll}
\hline Location & Record coordinates & Year & Reference \\
\hline Alexandra & $26^{\circ} 40^{\prime} 20^{\prime \prime} \mathrm{S}$ & 2004 & $\begin{array}{l}\text { Cobb \& } \\
\text { Headland, QLD }\end{array}$ \\
$153^{\circ} 06^{\prime} 49^{\prime \prime} \mathrm{E}$ & & Mullins (2009a) \\
Gold Coast, QLD & $27^{\circ} 56^{\prime} 09^{\prime \prime} \mathrm{S}$ & 2011 & Aston (2011a) \\
& $153^{\circ} 25^{\prime} 34^{\prime \prime} \mathrm{E}$ & & \\
Hastings & $28^{\circ} 21^{\prime} 37^{\prime \prime} \mathrm{S}$ & $2006-2011$ & Riek (2013a) \\
Point, NSW & $\begin{array}{l}153^{\circ} 34^{\prime} 45^{\prime \prime} \mathrm{E} \\
\text { Nelson Bay, NSW }\end{array}$ & & \\
& $32^{\circ} 43^{\prime} 13^{\prime \prime} \mathrm{S}$ & 2013 & This paper \\
& $152^{\circ} 08^{\prime} 39^{\prime \prime} \mathrm{E}$ & & \\
\hline
\end{tabular}

has been protected since 1983. Situated on a prominent point, this site has complex topography including a series of substantial ledges at various depths. While many habitats are similar to those at 'Pipeline', there are extensive areas of large sponges in the deeper sections (to $24 \mathrm{~m}$ ) (Coleman and Marsh 1997). 'Little Beach' is $450 \mathrm{~m}$ east of 'Fly Point' and comprises sandy substrate interspersed with seagrasses, sponges and gorgonians (Harasti et al., 2014).

Observations of sea slugs were made between 2009 and 2015 using SCUBA at the four dive sites. Many observations were made during recreational diving activities, as incidental sightings whilst undertaking other research or as part of broader research projects carried out by TRD and ML. Other observations were made as part of a Southern Cross University (SCU)/ Combined Hunter Underwater Research Group (CHUG) citizen-science project to document the diversity of sea slugs at three-monthly intervals over a two-year period (the Sea Slug Census).

Records of all species of sea slugs were collated from the authors' databases as well as from extensive

Table 3 Records of Thordisa tahala from Australian waters

\begin{tabular}{|c|c|c|c|}
\hline Location & Record coordinates & Year & Reference \\
\hline $\begin{array}{l}\text { Gneering } \\
\text { Shoals, QLD }\end{array}$ & $\begin{array}{l}26^{\circ} 38^{\prime} 54^{\prime \prime S} \\
153^{\circ} 10^{\prime} 58^{\prime \prime} \mathrm{E}\end{array}$ & 2014 & $\begin{array}{l}\text { Cobb \& } \\
\text { Mullins (2009d) }\end{array}$ \\
\hline $\begin{array}{l}\text { Gold Coast } \\
\text { Seaway, QLD }\end{array}$ & $\begin{array}{l}27^{\circ} 56^{\prime} 09^{\prime \prime S} \\
153^{\circ} 25^{\prime} 34^{\prime \prime} \mathrm{E}\end{array}$ & 2013 & $\begin{array}{l}\text { Aston (2011b), } \\
\text { Good (2015) }\end{array}$ \\
\hline $\begin{array}{l}\text { Brunswick } \\
\text { River, NSW }\end{array}$ & $\begin{array}{l}28^{\circ} 32^{\prime} 10^{\prime \prime S} \\
153^{\circ} 33^{\prime} 03^{\prime \prime} \mathrm{E}\end{array}$ & 2004-2014 & Riek (2013b) \\
\hline Nelson Bay, NSW & $\begin{array}{l}32^{\circ} 43^{\prime} 13^{\prime \prime S} \\
152^{\circ} 08^{\prime} 39^{\prime \prime} \mathrm{E}\end{array}$ & 2015 & This paper \\
\hline
\end{tabular}


Table 4 Records of Marionia pustulosa from Australian waters

\begin{tabular}{|c|c|c|c|}
\hline Location & $\begin{array}{l}\text { Record } \\
\text { coordinates }\end{array}$ & Year & Reference \\
\hline Dampier, WA & $\begin{array}{l}20^{\circ} 39^{\prime} 29^{\prime \prime S} \\
116^{\circ} 411^{\prime \prime} 59^{\prime \prime} \mathrm{E}\end{array}$ & 1990 & $\begin{array}{l}\text { (Coleman 2008, p. } \\
\text { 399) }\end{array}$ \\
\hline Port Curtis, QLD & $\begin{array}{l}23^{\circ} 49^{\prime} 00^{\prime \prime S} \\
151^{\circ} 16^{\prime} 25^{\prime \prime} \mathrm{E}\end{array}$ & 1929 & Odhner (1936) \\
\hline Noosa, QLD & $\begin{array}{l}26^{\circ} 22^{\prime} 39^{\prime \prime S} \mathrm{~S} \\
153^{\circ} 06^{\prime} 05^{\prime \prime} \mathrm{E}\end{array}$ & 2000 & $\begin{array}{l}\text { (Coleman 2008, p. } \\
\text { 399) }\end{array}$ \\
\hline Mooloolaba, QLD & $\begin{array}{l}26^{\circ} 40^{\prime} 34^{\prime \prime S} \\
153^{\circ} 07^{\prime} 09^{\prime \prime} \mathrm{E}\end{array}$ & 1997 & $\begin{array}{l}\text { (Coleman 2008, p. } \\
\text { 399) }\end{array}$ \\
\hline Flinders Reef, QLD & $\begin{array}{l}26^{\circ} 58^{\prime} 47^{\prime \prime S} \\
153^{\circ} 29^{\prime} 06^{\prime \prime E}\end{array}$ & 2009 & $\begin{array}{l}\text { Cobb \& Mullins } \\
\text { (2009b) }\end{array}$ \\
\hline Mud Island, QLD & $\begin{array}{l}27^{\circ} 20^{\prime} 32^{\prime \prime S} \\
153^{\circ} 14^{\prime \prime} 15^{\prime \prime} \mathrm{E}\end{array}$ & 1972 & Thompson (1972) \\
\hline $\begin{array}{l}\text { North Solitary } \\
\text { Island, NSW }\end{array}$ & $\begin{array}{l}29^{\circ} 55^{\prime \prime} 18^{\prime \prime S} \\
153^{\circ} 23^{\prime} 14^{\prime \prime} \mathrm{E}\end{array}$ & 1989 & $\begin{array}{l}\text { Australian Museum } \\
\text { (1989) }\end{array}$ \\
\hline Nelson Bay, NSW & $\begin{array}{l}32^{\circ} 43^{\prime} 13^{\prime \prime S} \\
152^{\circ} 08^{\prime} 39^{\prime \prime} \mathrm{E}\end{array}$ & $\begin{array}{l}1988,1996, \\
2015\end{array}$ & $\begin{array}{l}\text { Carol Buchanan, this } \\
\text { paper. }\end{array}$ \\
\hline
\end{tabular}

photographic material from key underwater photographers. Species observations that had been reported online (in Nudi Pixel or the Sea Slug Forum) were considered as published observations.

\section{Results}

\section{Systematics}

Order CEPHALASPIDEA Fischer, 1887

Family AGLAJIDAE Pilsbry, 1895 (1847)

Genus Philinopsis Pease, 1860

Philinopsis orientalis (Baba, 1949)

Synonym: Aglaja orientalis Baba, 1949

This distinctive aglajid was originally named Aglaja orientalis by Baba (1949) from specimens found off

Table 5 Records of Trinchesia ornata from Australian waters

\begin{tabular}{|c|c|c|c|}
\hline Location & Record coordinates & Year & Reference \\
\hline $\begin{array}{l}\text { Heron } \\
\text { Island, } \\
\text { QLD }\end{array}$ & $\begin{array}{l}23^{\circ} 27^{\prime} 04^{\prime \prime S} \\
151^{\circ} 55^{\prime} 177^{\prime \prime E}\end{array}$ & 1998-1994 & $\begin{array}{l}\text { (Marshall \& } \\
\text { Willan 1999, p. 141) }\end{array}$ \\
\hline $\begin{array}{l}\text { Heron } \\
\text { Island, } \\
\text { QLD }\end{array}$ & $\begin{array}{l}23^{\circ} 27^{\prime} 04^{\prime \prime S} \\
151^{\circ} 55^{\prime} 17^{\prime \prime} \mathrm{E}\end{array}$ & 1994 & $\begin{array}{l}\text { Museum } \\
\text { Victoria (1980) }\end{array}$ \\
\hline $\begin{array}{l}\text { Gneering } \\
\text { Shoals, } \\
\text { QLD }\end{array}$ & $\begin{array}{l}26^{\circ} 38^{\prime} 54^{\prime \prime S} \mathrm{~S} \\
153^{\circ} 10^{\prime} 58^{\prime \prime} \mathrm{E}\end{array}$ & 2004 & $\begin{array}{l}\text { MAGNT } \\
\text { 2011) }\end{array}$ \\
\hline $\begin{array}{l}\text { Alexandra } \\
\text { Headland, } \\
\text { QLD }\end{array}$ & $\begin{array}{l}26^{\circ} 40^{\prime} 20^{\prime \prime S} \\
153^{\circ} 06^{\prime} 49^{\prime \prime} \mathrm{E}\end{array}$ & 2004 & $\begin{array}{l}\text { (Coleman } \\
\text { 2008, p. 379) }\end{array}$ \\
\hline $\begin{array}{l}\text { Byron Bay, } \\
\text { NSW }\end{array}$ & $\begin{array}{l}28^{\circ} 36^{\prime} 41^{\prime \prime S} \\
153^{\circ} 37^{\prime} 46^{\prime \prime} \mathrm{E}\end{array}$ & 1992 & $\begin{array}{l}\text { (Coleman } \\
\text { 2008, p. 379) }\end{array}$ \\
\hline Nelson Bay, NSW & $\begin{array}{l}32^{\circ} 43^{\prime} 13^{\prime \prime S} \\
152^{\circ} 08^{\prime} 39^{\prime \prime} \mathrm{E}\end{array}$ & 2013 & This paper \\
\hline
\end{tabular}

Table 6 Records of Trinchesia puellula from Australian waters

\begin{tabular}{llll}
\hline Location & Record coordinates & Year & Reference \\
\hline Gneering Shoals, & $26^{\circ} 38^{\prime} 54^{\prime \prime} S 153^{\circ} 10^{\prime}$ & 2008 & Cobb \& Mullins \\
QLD & $58^{\prime \prime} \mathrm{E}$ & & (2008) \\
Nelson Bay, NSW & $32^{\circ} 43^{\prime} 13^{\prime \prime} S 152^{\circ} 08^{\prime}$ & 2013 & This paper \\
& $39^{\prime \prime} \mathrm{E}$ & & \\
\hline
\end{tabular}

Kurosaki and Hayama in Sagami Bay, Japan. Baba expressed doubts about that generic location in correspondence with RCW. It was recently transferred into the genus Philinopsis (Camacho-García et al. 2013). Since then, it has been found at several locations throughout the Indo-Pacific including La Reunion and New Caledonia (Rudman, 2003f). It is distinguished by the presence of numerous scattered white spots and areas of opaque white marks containing smaller yellow lines and spots that form a line across the head and transversely across the mid-notum, and a patch on the tail (Gosliner et al., 2008, p. 42). In Australian waters, there are published records of $P$. orientalis from Rottnest Island, WA (Wells \& Bryce, 2000, p. 31-32) and from the (northern) GBR (Marshall \& Willan, 1999, p. 171) (Table 1). An observation of a $15 \mathrm{~mm}$ specimen at $8 \mathrm{~m}$ depth on 14 February, 2015 at 'Fly Point' (Fig. 2a) extends the east coast range for this species by $2,246 \mathrm{~km}$ to central NSW.

\section{Order NUDIBRANCHIA Cuvier, 1817 \\ Family DISCODORIDIDAE Bergh, 1891 \\ Genus Jorunna Bergh, 1876 \\ Jorunna ramicola M. C. Miller, 1996}

Jorunna ramicola was described first from New Zealand (Miller, 1996); however, it has subsequently been found widely in the Indo-Pacific Ocean with a distribution that includes Madagascar, Japan, Philippines and Papua New Guinea (Camacho-García \& Gosliner, 2008; Gosliner et al. 2008) (Note that this assumption of a single species has not been tested genetically and species of Jorunna are notoriously difficult to identify morphologically). As with many discodorids, this species exhibits protective resemblance (Behrens et al., 2005) by mimicking the surface pattern and colour of its host sponge (Callyspongia) and is thus well camouflaged. In Australia, this

Table 7 Records of Facelina rhodopos from Australian waters

\begin{tabular}{|c|c|c|c|}
\hline Location & Record coordinates & Year & Reference \\
\hline Lizard Island, QLD & $\begin{array}{l}14^{\circ} 40^{\prime} 43^{\prime \prime} \mathrm{S} 145^{\circ} 88^{\prime} \\
13^{\prime \prime} \mathrm{E}\end{array}$ & 2002 & $\begin{array}{l}\text { Australian Museum } \\
(2002)\end{array}$ \\
\hline $\begin{array}{l}\text { Hastings Point, } \\
\text { NSW }\end{array}$ & $\begin{array}{l}28^{\circ} 21^{\prime} 37^{\prime \prime} \mathrm{S} 153^{\circ} 34^{\prime} \\
45^{\prime \prime} \mathrm{E}\end{array}$ & 2005 & Riek (2013c) \\
\hline $\begin{array}{l}\text { Sandy Beach, } \\
\text { NSW }\end{array}$ & $\begin{array}{l}30^{\circ} 08^{\prime} 44^{\prime \prime} \mathrm{S} 153^{\circ} 12^{\prime} \\
08^{\prime \prime} \mathrm{E}\end{array}$ & 2014 & Pers. obs. (M. Nimbs) \\
\hline Nelson Bay, NSW & $\begin{array}{l}32^{\circ} 43^{\prime} 13^{\prime \prime} \mathrm{S} 152^{\circ} 08^{\prime} \\
39^{\prime \prime} \mathrm{E}\end{array}$ & 2014 & This paper \\
\hline
\end{tabular}


Table 8 Records of Sakuraeolis nungunoides from Australian waters

\begin{tabular}{|c|c|c|c|}
\hline Location & $\begin{array}{l}\text { Record } \\
\text { coordinates }\end{array}$ & Year & Reference \\
\hline $\begin{array}{l}\text { Mudjimba Island, } \\
\text { QLD }\end{array}$ & $\begin{array}{l}26^{\circ} 36^{\prime} 52^{\prime \prime} \mathrm{S} 153^{\circ} 06^{\prime} \\
53^{\prime \prime} \mathrm{E}\end{array}$ & 2012 & $\begin{array}{l}\text { Cobb \& Mullins } \\
\text { (2012b) }\end{array}$ \\
\hline Gold Coast, QLD & $\begin{array}{l}27^{\circ} 56^{\prime} 09^{\prime \prime} \mathrm{S} 153^{\circ} 25^{\prime} \\
34^{\prime \prime} \mathrm{E}\end{array}$ & 2012 & Good (2012) \\
\hline $\begin{array}{l}\text { Brunswick River, } \\
\text { NSW }\end{array}$ & $\begin{array}{l}28^{\circ} 32^{\prime} 10^{\prime \prime S} 153^{\circ} 33^{\prime} \\
03^{\prime \prime} \mathrm{E}\end{array}$ & 2004 & Riek (2013d) \\
\hline Nelson Bay, NSW & $\begin{array}{l}32^{\circ} 43^{\prime} 13^{\prime \prime} \mathrm{S} 152^{\circ} 08^{\prime} \\
39^{\prime \prime} \mathrm{E}\end{array}$ & $\begin{array}{l}2013- \\
2015\end{array}$ & This paper \\
\hline
\end{tabular}

species has been recorded several times from subtropical QLD and northern NSW (Table 2). Prior to our observation of a $12 \mathrm{~mm}$ individual at $6 \mathrm{~m}$ depth from the 'Pipeline' on 30 November, 2013 (Fig. 2b), the southernmost record of $J$. ramicola was at Hastings Point, NSW, on 27 April, 2010 (Riek, 2013a). The Nelson Bay record extends the range south by $500 \mathrm{~km}$.

Genus Thordisa Bergh, 1877

Thordisa tahala Chan \& Gosliner, 2007

The original description of Thordisa tahala was based on descriptions from preserved specimens from Madagascar, Indonesia and the Marshall Islands (Chan \& Gosliner, 2007), indicating a wide Indo-Pacific distribution (Gosliner et al., 2008). The specific name was derived from Malagasy (tahala = ridge) for the network of raised ridges on the dorsum and, in conjunction with its colour, the network pattern distinguishes T. tahala from any other species within the genus (Chan \& Gosliner, 2007). The previously known range of $T$. tahala in
Australian waters was a $210 \mathrm{~km}$ section of coast from the Gneering Shoals in southern QLD to the Brunswick River, NSW (Table 3). This observation on 22 January, 2015 of a $60 \mathrm{~mm}$ specimen (Fig. 2c) at $8 \mathrm{~m}$ depth, at night, from 'Little Beach' extends the east coast range for this species by $480 \mathrm{~km}$.

\section{Family TRITONIIDAE Lamarck, 1809 \\ Genus Marionia Vayssière, 1877 \\ Marionia pustulosa Odhner, 1936}

Marionia pustulosa is a moderately large sea slug which, when amongst its host octocoral, is camouflaged using a complex pattern of reticulated lines, various muted colours and many elevated papillae on its dorsum, oral veil and rhinophoral sheaths. This dendronotid nudibranch appears endemic to Australia and was first described by Odhner from a specimen sourced from Port Curtis, QLD, in 1929 and later redescribed by Thompson (1972) using a live specimen found in Moreton Bay, QLD, in 1972. It has been recorded from Dampier, WA, Noosa, QLD (Coleman, 2008), and North Solitary Island, NSW (Australian Museum, 1989), amongst other locations (Table 4). This species was photographed by Carol Buchanan in Nelson Bay in March 1988 and again in March 1996; however, these observations remained unpublished. Those earlier observations, and another of a $35 \mathrm{~mm}$ specimen (Fig. 2d) in $6 \mathrm{~m}$ of water at 'Seahorse Gardens' on 7 March, 2015 increase the southern range of $M$. pustulosa by $332 \mathrm{~km}$.

Family TERGEPEDIDAE Bergh, 1889

Genus Trinchesia Ihering, 1879
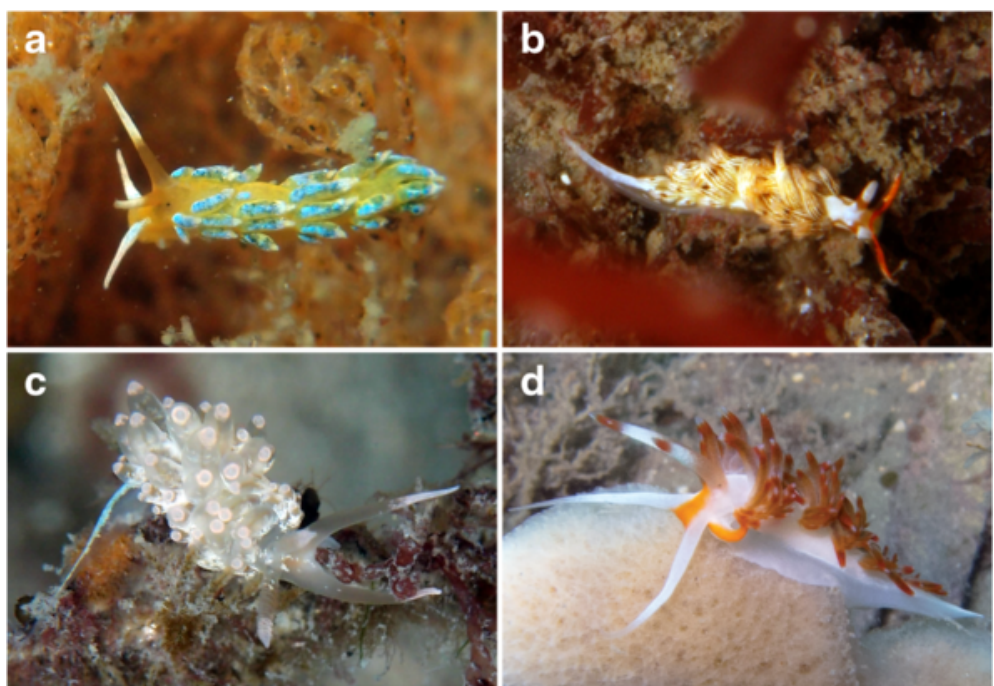

Fig. 3 a. Trinchesia ornata. Little Beach, 8 December, 2013. b. Trinchesia puellula. Little Beach, 11 September, 2013. c. Facelina rhodopos. Little Beach, 15 December, 2014. d. Sakuraeolis nungunoides. Fly Point, 23 August, 2013. Photos: a, b, c, Tom Davis; d, Nicola Davis 
Table 9 Records of Kaloplocamus peludo from Australian waters

\begin{tabular}{llll}
\hline Location & Record coordinates & Year & Reference \\
\hline Heron Island, QLD & $23^{\circ} 27^{\prime} 04^{\prime \prime} \mathrm{S} 151^{\circ} 55^{\prime}$ & 2007 & Rudman (2007) \\
$17^{\prime \prime E}$ & & & \\
& $26^{\circ} 46^{\prime} 02^{\prime \prime} \mathrm{S} 153^{\circ} 09^{\prime}$ & 2014 & Cobb \& Mullins \\
Currimundi Reef, & $34^{\prime \prime E}$ & & (2012a) \\
QLD & $32^{\circ} 43^{\prime} 13^{\prime \prime} \mathrm{S} 152^{\circ} 08^{\prime}$ & 2014 & This paper \\
Nelson Bay, NSW & $39^{\prime \prime E}$ & & \\
\hline
\end{tabular}

Trinchesia ornata (Baba, 1937)

Synonyms: Catriona ornata (Baba, 1937), Cuthona ornata Baba, 1937

Trinchesia ornata is recognised by its orange body with blue and yellow cerata (Gosliner et al., 2008) and by its obligate predation of thecate hydroids (Gosliner, 1987; Marshall \& Willan, 1999). Although originally described from Tomioka in Japan (Baba, 1937), its range extends from South Africa (Gosliner, 1987, pp. 20, 116), La Réunion (Nudi Pixel, 2011d) to Japan, Hong Kong (Gosliner et al., 2008), Indonesia, Thailand, Philippines, Palau (Nudi Pixel, 2011e) and Australia (Marshall \& Willan, 1999; Coleman, 2008). In Australia, T. ornata has previously been recorded from various locations from Heron Island in the southern GBR to Byron Bay, NSW (Table 5). It is rather difficult to see underwater, but is assumed to be quite common. On 8 December, 2013 a $5 \mathrm{~mm}$ specimen was observed at 'Little Beach' on rocky reef at 8 $\mathrm{m}$ deep (Fig. 3a), $475 \mathrm{~km}$ south of its previously reported southernmost distributional limit.

Trinchesia puellula Baba, 1955
Synonyms: Cratena puellula Baba, 1955, Catriona puellula (Baba, 1955), Cuthona puellula (Baba, 1955)

Baba (1955) described this species from a single 10 $\mathrm{mm}$ animal from Hayama, Sagami Bay, Japan. It is characterised by the presence of orange markings on both the rhinophores and oral tentacles, vertical white lines on the cerata, and the black speckled appearance of the digestive gland within the cerata (Rudman, 2002d). It was later reported from California (Marcus, Er. 1961) and then from Australia in 2008 at Gneering Shoals, QLD (Table 6), implying a broad distribution across the Pacific Ocean, most likely facilitated by shipping. Little is known of the biology and ecology of this species with few observations recorded outside Japanese waters. Our recent observation of a $5 \mathrm{~mm}$ specimen on sand at 'Little Beach' on 11 September, 2013 at $7 \mathrm{~m}$ depth constitutes a southward range extension of $680 \mathrm{~km}$ (Fig. 3b).

Family FACELINIDAE Alder \& Hancock, 1855

Genus Facelina Alder \& Hancock, 1855

Facelina rhodopos Yonow, 2000

Synonym: Pruvotfolia rhodopos (Yonow, 2000)

This aeolid has an Indo-Pacific distribution from the Red Sea (Yonow, 2000), through La Réunion (Bidgrain, 2006), Kenya, Philippines, Indonesia (Nudi Pixel, 2011a) and Malaysia (Rudman, 2002a) to Australia. Facelina rhodopos bears clusters of cerata tipped with pink/peach rings and an opaque longitudinal metapodial line which are diagnostic of the species (Yonow, 2000). In Australian waters, it has been found in the northern GBR at Lizard Island, QLD, and in northern NSW from Hastings Point

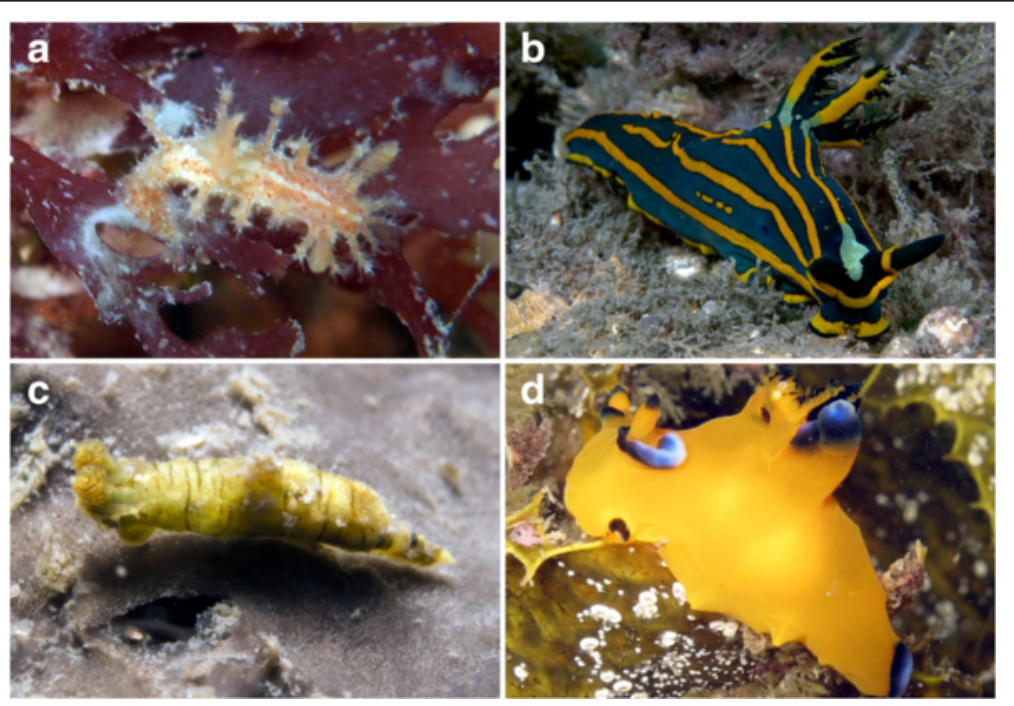

Fig. 4 a. Kaloplocamus peludo. Fly Point, 11 September, 2014. b. Tambja victoriae. Fly Point, 20 February, 2010. c. Polycera risbeci. Pipeline, 23 November, 2014. d. Thecacera pacifica. Fly Point, 6 December, 2014. Photos: a, Tom Davis; b, Roxanne Streatfeild; c, David Harasti; d, Meryl Larkin 
Table 10 Records of Tambja victoriae from Australian waters

\begin{tabular}{llll}
\hline Location & Record coordinates & Year & Reference \\
\hline Hibernia Passage, QLD & $12^{\circ} 47^{\prime} 54^{\prime \prime} \mathrm{S} 143^{\circ} 48^{\prime} 47^{\prime \prime} \mathrm{E}$ & 1981 & Australian Museum (1981) \\
Swain Reefs, QLD & $21^{\circ} 14^{\prime} 22^{\prime \prime} \mathrm{S} 151^{\circ} 50^{\prime} 48^{\prime \prime} \mathrm{E}$ & 1985 & Australian Museum (1985) \\
North West Reef, QLD & $23^{\circ} 14^{\prime} 54^{\prime \prime} \mathrm{S} 151^{\circ} 46^{\prime} 41^{\prime \prime} \mathrm{E}$ & 1983 & Australian Museum (1983) \\
Heron Island, QLD & $23^{\circ} 27^{\prime} 04^{\prime \prime} \mathrm{S} 151^{\circ} 55^{\prime} 17^{\prime \prime} \mathrm{E}$ & $1973-2008$ & $\begin{array}{l}\text { Nudi Pixel (2003); Rudman (2005); } \\
\text { (Coleman 2008, p. 369); Nudi Pixel (2008d) }\end{array}$ \\
Mooloolaba, QLD & $26^{\circ} 39^{\prime} 37^{\prime \prime} \mathrm{S} 153^{\circ} 08^{\prime} 08^{\prime \prime} \mathrm{E}$ & 2003 & Cobb \& Mullins (2003) \\
Gold Coast Seaway, QLD & $27^{\circ} 56^{\prime} 09^{\prime \prime} \mathrm{S} 153^{\circ} 25^{\prime} 34^{\prime \prime E}$ & 2014 & Aston (2014) \\
Nelson Bay, NSW & $32^{\circ} 43^{\prime} 13^{\prime \prime} \mathrm{S} 152^{\circ} 08^{\prime} 39^{\prime \prime} \mathrm{E}$ & 2010 & This paper \\
\hline
\end{tabular}

and Sandy Beach (Table 7). On 15 December, 2014 at 'Little Beach', we observed a $15 \mathrm{~mm}$ specimen at $7 \mathrm{~m}$ depth on a rocky reef (Fig. 3c) at night. This record extends the east coast range southward by $300 \mathrm{~km}$.

Genus Sakuraeolis Baba, 1965

Sakuraeolis nungunoides Rudman, 1980

Sakuraeolis nungunoides was originally described from Tanzania in tropical eastern Africa. Its range has subsequently been found to extend into the Indo-Pacific reaching Malaysia, the Philippines, New Caledonia and Australia (Rudman, 2003d). This species bears long, transparent cerata which are frequently held erect; each has a subapical orange band. A median orange band extends between the base of the rhinophores and the oral tentacles. The rhinophores are long, tapering, translucent and tipped in orange (Rudman, 2003d). When irritated, S. nungunoides straightens and 'bristles' each ceras which, according to Rudman, is similar to the behaviour of a porcupine (Swahili = Nungunungu), for which this species is named. In Australia, S. nungunoides has been recorded from the Sunshine Coast (Mudjimba Island), QLD, to the Brunswick River, NSW (Table 8). The observation of a $35 \mathrm{~mm}$ specimen at 'Fly Point' on 23 August, 2013 at $14 \mathrm{~m}$, and two additional individuals 'Little Beach' at $11 \mathrm{~m}$ deep on 2 February, 2015 (Fig. 3d) extend its southern range by $475 \mathrm{~km}$.

Table 11 Records of Polycera risbeci from Australian waters

\begin{tabular}{|c|c|c|c|}
\hline Location & $\begin{array}{l}\text { Record } \\
\text { coordinates }\end{array}$ & Year & Reference \\
\hline $\begin{array}{l}\text { Heron Island, } \\
\text { QLD }\end{array}$ & $\begin{array}{l}23^{\circ} 27^{\prime} 04^{\prime \prime} \mathrm{S} 151^{\circ} 55^{\prime} \\
17^{\prime \prime} \mathrm{E}\end{array}$ & $\begin{array}{l}1981- \\
1994\end{array}$ & $\begin{array}{l}\text { (Marshall \& Willan 1999, } \\
\text { p. 53) }\end{array}$ \\
\hline $\begin{array}{l}\text { Mooloolaba, } \\
\text { QLD }\end{array}$ & $\begin{array}{l}26^{\circ} 40^{\prime} 34^{\prime \prime} \mathrm{S} 153^{\circ} 07^{\prime} \\
09^{\prime \prime} \mathrm{E}\end{array}$ & 2009 & Cobb \& Mullins (2009c) \\
\hline $\begin{array}{l}\text { Hastings Point, } \\
\text { NSW }\end{array}$ & $\begin{array}{l}28^{\circ} 21^{\prime} 37^{\prime \prime} \mathrm{S} 153^{\circ} 34^{\prime} \\
45^{\prime \prime} \mathrm{E}\end{array}$ & $\begin{array}{l}2006 \\
2013\end{array}$ & Riek (2013e) \\
\hline $\begin{array}{l}\text { Nelson Bay, } \\
\text { NSW }\end{array}$ & $\begin{array}{l}32^{\circ} 43^{\prime} 13^{\prime \prime S} 152^{\circ} 08^{\prime} \\
39^{\prime \prime} \mathrm{E}\end{array}$ & 2014 & This paper \\
\hline
\end{tabular}

Family POLYCERIDAE Alder \& Hancock, 1845

Genus Kaloplocamus Bergh, 1880

Kaloplocamus peludo Vallès \& Gosliner, 2006

This polycerid feeds on arborescent bryozoans and possesses numerous dorsal papillae (Gosliner \& Vallès, 2006) that afford it a strong resemblance to its prey (Gosliner et al., 2008, p. 105). Kaloplocamus peludo has a wide Indo-Pacific distribution and, in Australia, has been recorded at Heron Island, QLD, in 2007, and more recently at Currimundi Reef, QLD in 2014 (Table 9). This observation of a $10 \mathrm{~mm}$ specimen at 'Fly Point' on 11 September 2014 at $7 \mathrm{~m}$ depth represents a $640 \mathrm{~km}$ southward range extension (Fig. 4a).

\section{Genus Tambja Burn, 1962}

Tambja victoriae Pola, Cervera \& Gosliner, 2005

This species is distinguished from other Tambja species by the presence of dark blue rhinophores surrounded by dark blue rhinophoral sheaths edged in yellow (Gosliner et al., 2008, p. 118) and is known to feed on arborescent bryozoans. Its distribution is

Table 12 Records of Thecacera pacifica from Australian waters

\begin{tabular}{|c|c|c|c|}
\hline Location & Record coordinates & Year & Reference \\
\hline Christmas Island & $\begin{array}{l}10^{\circ} 30^{\prime} 08^{\prime \prime S} 105^{\circ} 40^{\prime} \\
59^{\prime \prime} \mathrm{E}\end{array}$ & 1987 & Rudman (2002c) \\
\hline $\begin{array}{l}\text { Bynoe Harbour, } \\
\text { NT }\end{array}$ & $\begin{array}{l}13^{\circ} 39^{\prime} 511^{\prime \prime} \mathrm{S} 130^{\circ} 32^{\prime} \\
12^{\prime \prime} \mathrm{E}\end{array}$ & 2003 & MAGNT (2003) \\
\hline $\begin{array}{l}\text { Kendrew Island, } \\
\text { WA }\end{array}$ & $\begin{array}{l}20^{\circ} 28^{\prime} 41^{\prime \prime S} 116^{\circ} 32^{\prime} \\
23^{\prime \prime} \mathrm{E}\end{array}$ & 1972 & $\begin{array}{l}\text { Museum Victoria } \\
\text { (1975a) }\end{array}$ \\
\hline Tweed River, NSW & $\begin{array}{l}28^{\circ} 10^{\prime} 18^{\prime \prime} \mathrm{S} 153^{\circ} 32^{\prime} \\
57^{\prime \prime} \mathrm{E}\end{array}$ & 2013 & Aston (2013) \\
\hline $\begin{array}{l}\text { Rottnest Island, } \\
\text { WA }\end{array}$ & $\begin{array}{l}31^{\circ} 59^{\prime} 19^{\prime \prime} \mathrm{S} 115^{\circ} 30^{\prime} \\
10^{\prime \prime E}\end{array}$ & 2011 & Nudi Pixel (2011c) \\
\hline Nelson Bay, NSW & $\begin{array}{l}32^{\circ} 43^{\prime} 13^{\prime \prime} \mathrm{S} 152^{\circ} 08^{\prime} \\
39^{\prime \prime} \mathrm{E}\end{array}$ & 2014 & This paper \\
\hline Esperance, WA & $\begin{array}{l}33^{\circ} 21^{\prime} 00^{\prime \prime} \mathrm{S} 121^{\circ} 53^{\prime} \\
00^{\prime \prime} \mathrm{E}\end{array}$ & 1985 & Clay Bryce, this paper \\
\hline Albany, WA & $\begin{array}{l}35^{\circ} 04^{\prime} 43^{\prime \prime S} 117^{\circ} 57^{\prime} \\
03^{\prime \prime} \mathrm{E}\end{array}$ & 2010 & Nudi Pixel (2010d) \\
\hline
\end{tabular}


Table 13 Sea slug species with a southern distribution limit on the eastern Australian coast at Port Stephens, NSW

\begin{tabular}{|c|c|c|c|}
\hline Order & Species & Published record & Reference \\
\hline \multirow[t]{2}{*}{ Cephalaspidea } & Philinopsis reticulata & 2004 & NudiPixel (2004) \\
\hline & Haminoea cymbalum & 1980 & Australian Museum (1980) \\
\hline \multirow[t]{2}{*}{ Sacoglossa } & Lobiger viridis & 2009 & Rudman (2009) \\
\hline & Polybranchia orientalis & 1999 & Rudman (1999e) \\
\hline Pleurobranchomorpha & Pleurobranchus forskalii & 2012 & Nudi Pixel (2012) \\
\hline \multirow[t]{25}{*}{ Nudibranchia } & Nembrotha purpureolineata & 1999 & Rudman (1999b) \\
\hline & Nembrotha rosannulata & 2004 & Rudman (2008) \\
\hline & Polycera melanosticta & 2014 & MAGNT (2014) \\
\hline & Okenia hallucigenia & 1986 & Australian Museum (1986) \\
\hline & Okenia harastii & 2013 & Pola et al., 2014 \\
\hline & Okenia purpurata & 2000 & Rudman (2006b) \\
\hline & Okenia vena & 2000 & Rudman (2006c) \\
\hline & Aegires incusus & 2009 & Nudi Pixel (2009a) \\
\hline & Ceratosoma tenue & 2008 & Nudi Pixel (2008a) \\
\hline & Chromodoris striatella & 2000 & Rudman (2000a) \\
\hline & Goniobranchus albonares & 1998 & Rudman (1998a) \\
\hline & Goniobranchus verrieri & 2003 & Rudman (2003a) \\
\hline & Hypselodoris tryoni & 2010 & Nudi Pixel (2010c) \\
\hline & Noumea laboutei & 2008 & Nudi Pixel (2008c) \\
\hline & Noumea varians & 1999 & Rudman (1999d) \\
\hline & Thorunna florens & 2002 & Rudman (2003e) \\
\hline & Platydoris ellioti & 2010 & Nudi Pixel (2010b) \\
\hline & Sebadoris fragilis & 1997 & Rudman (1998b) \\
\hline & Dermatobranchus dendronephthyphagus & 2004 & Rudman (2004a) \\
\hline & Crosslandia viridis & 1999 & Rudman (1999a) \\
\hline & Notobryon wardi & 1999 & Rudman (1999c) \\
\hline & Eubranchus ocellatus & 2010 & Nudi Pixel (2010a) \\
\hline & Cuthona yamasui & 2008 & Nudi Pixel (2009b) \\
\hline & Trinchesia sibogae & 1999 & Rudman (2003b) \\
\hline & Cerberilla ambonensis & 2010 & Rudman (2010) \\
\hline
\end{tabular}

thought to be restricted to the western Pacific Ocean, extending from the Philippines to Papua New Guinea and eastern Australia (Rudman, 2005; Gosliner et al., 2008). However, this has not been tested genetically. Australian records indicate a distribution spanning the northern GBR to the Gold Coast in southeastern QLD (Table 10). The observation of a $30 \mathrm{~mm}$ individual at 'Fly Point', on 20 February, 2010 at $7 \mathrm{~m}$ depth constitutes a southward range extension of $700 \mathrm{~km}$ (Fig. 4b).

Genus Polycera Cuvier, 1816

Polycera risbeci Odhner, 1941

This small polycerid is distinguished by the presence of several transverse, broken, brown lines across the body (Marshall \& Willan 1999, p. 53; Gosliner et al.,
2008, p. 102). The gills are pale yellow and the rhinophores are large. Polycera risbeci occurs in the tropical Indian and western Pacific Oceans (Gosliner et al., 2008, p. 102) with an Australian distribution extending from Heron Island, QLD, to northern NSW (Table 11). On 23 November, 2014 an individual measuring approximately $4 \mathrm{~mm}$ was observed at the 'Pipeline' at $5 \mathrm{~m}$ (Fig. 4c) extending its southern range by $665 \mathrm{~km}$.

Genus Thecacera Fleming, 1828

Thecacera pacifica (Bergh, 1884)

Synonyms: Ohola pacifica Bergh, 1884, Thecacera inhacae MacNae, 1958

Thecacera pacifica has an orange body with black, blue and white tips to the tail and both the extra- 
rhinophoral and extra-branchial appendages (Gosliner et al., 2008, p. 109). This well-known species has become colloquially known as the Pikachu sea slug in Japan and on social media after its similarity to a popular Pokémon toy (Rudman, 2003c; Simonitch, 2012). It has a wide distribution from southern Africa, the Red Sea, Japan, Australia, Hawai'i and the Gulf of Mexico (Gosliner et al., 2008, p. 109), though this has not been tested genetically. The extension of this species into the Gulf of Mexico may be the result of dispersal via shipping as is the case for its congener T. pennigera (Willan, 1976). Although T. pacifica has been found several times in WA, including observations as far south as Esperance in 1985 and Albany in 2010, it has been recorded only once on the east coast at the Tweed River, NSW, in 2013 (Table 12). An observation of a $30 \mathrm{~mm}$ individual at 'Fly Point' at $7 \mathrm{~m}$ depth on 6 December, 2014 extends its southern range by $685 \mathrm{~km}$ (Fig. 4d).

\section{Discussion}

Preliminary results from a biogeographic study of sea slug distribution on the NSW coast (by MN) record 313 species for Port Stephens. Of those, 30 species have not been found further south on the east coast (Table 13).

As a well-studied sea slug 'hot spot', there are extensive records for Port Stephens that clearly indicate a sustained high species richness. These observations of 12 heterobranch sea slugs substantially (i.e., by distances greater than $300 \mathrm{~km}$ ) south of their previously reported range provide support for other observations of range extensions of other taxa during the last decade. For example, the tropical stichodactylid actinian Stichodactyla haddoni (Saville-Kent, 1893), host for three species of tropical commensal shrimps, was recently reported from Port Stephens and Sydney Harbour (Scott et al., 2015). Other documented range extensions on Australia's eastern coast include four heterobranch sea slugs on the mid-north coast of NSW (Nimbs et al. 2015) and a number of intertidal mollusc species in eastern Tasmania (Pitt et al., 2010).

There are two important additional points to make about the range extensions to Port Stephens that have been documented here. Firstly, almost half of the species were found only as juveniles suggesting that, whilst they may recruit to the Port, they may not survive to adulthood or to form breeding populations there. Indeed, the fate of juveniles found at the limit of their geographic range is a topic requiring further study. Secondly, whilst our primary hypothesis is that the range extensions result from climate change related processes, the alternate hypothesis of greater sampling effort needs to be considered. Port Stephens is progressively attracting greater attention from scientists and also from citizen scientists who are becoming more experienced in differentiating between similar heterobranch taxa, particularly the smaller species. Citizen science participation has expanded substantially over the past few years through programs such as the Sea Slug Census and the Nelson Bay Nudi Festival. Therefore, it is likely that at least some of the range extensions reported here may be of species that were overlooked in the past.

\section{Acknowledgements}

The authors extend their gratitude to: Denis Riek for detailed information regarding the large number of species he has observed on the Tweed-Byron coast; Deb Aston for her generosity in time and information regarding sea slugs found in the Gold Coast region over many years; and Carol Buchanan for generously allowing the first author full access to her vast collection of heterobranch photographs and personal knowledge gathered over several decades. Thanks are also due to the following photographers for permission to use their images: Matt Doyle for his photograph of Philinopsis orientalis; Roxanne Streatfeild for her photograph of Tambja victoriae; Nicola Davis for her photograph of Sakuraeolis nungunoides; and Kristine O'Keefe for her photograph of Marionia pustulosa. We also acknowledge all of the participants in the Sea Slug Census program for their eager participation in the series of events from 2013 to the present. Kathryn James provided professional design for the photographic figures. Robert Burn kindly checked his copies of Bergh's publications. This paper was prepared from data collected as part of a BSC (Hons) research project by MN, a PhD project by TRD, and a MSc project by ML. Funding and in-kind support was provided by Southern Cross University and NSW DPI (Fisheries). The authors also thank three anonymous reviewers for their constructive comments that helped improve the manuscript.

\section{Authors' contributions}

MN collated occurrence records and with RCW and SDAS wrote the manuscript. ML, TD, DH and SDAS carried out fieldwork and documented species occurrence. RCW identified the animals from photographs. All authors read and approved the final manuscript.

\section{Competing interests}

The authors declare that they have no competing interests.

\section{Author details}

${ }^{1}$ National Marine Science Centre, Southern Cross University, P.O. Box 4321, Coffs Harbour, NSW 2450, Australia. ${ }^{2}$ Marine Ecology Research Centre, Southern Cross University, Lismore, NSW 2456, Australia. ${ }^{3}$ NSW Department of Industry, Fisheries NSW, Marine Ecosystems Research, Nelson Bay, NSW 2315, Australia. ${ }^{4}$ Museum and Art Gallery of the Northern Territory, G.P.O. Box 4646, Darwin, NT 0801, Australia.

Received: 7 September 2015 Accepted: 2 December 2015 Published online: 01 July 2016

\section{References}

Aston D. Jorunna ramicola. 2011a. Retrieved from http://www. astonunderwaterimages.com/keyword/jorunna/ on 08 July 2015

Aston D. Tambja victoriae. 2014. Retrieved from http://www. astonunderwaterimages.com/GoldCoastSeaway/Nudibranchs-Gold-Coast/iz55jFPK on 08 July 2015.

Aston D. Thecacera Pacifica. 2013. Retrieved from http://www. astonunderwaterimages.com/GoldCoastSeaway/Nudibranchs-Gold-Coast/ip6ZQJmT on 02 August 2015.

Aston D. Thordisa Tahala. 2011 b. Retrieved from http://www. astonunderwaterimages.com/Nudibranch/The-Latest-Nudibranch-finds/isBCgxq7 on 08 July 2015.

Australian Museum. Occurrence record: Malacology:C.125185 Haminoea cymbalum. 1980. Retrieved from http://biocache.ala.org.au/occurrences/ 16adc431-8cc4-400c-8b93-0359a497299a on 05 August 2015.

Australian Museum. Occurrence record: Malacology:C.132469.001 Tambja victoriae. 1981. Retrieved from http://biocache.ala.org.au/occurrences/22834a8a-eb8a4852-9697-b32487f13823 on 10 July 2015. 
Australian Museum. Occurrence record: Malacology:C.140390.001 Tambja victoriae. 1983. Retrieved from http://biocache.ala.org.au/occurrences/ e3d45547-f777-4313-9521-ba3ec25461b3 on 10 July 2015.

Australian Museum. Occurrence record: Malacology:C.145059.001 Tambja victoriae. 1985. Retrieved from http://biocache.ala.org.au/occurrences/3c36bb5e-86374419-a4a0-32f572c092ba on 10 July 2015.

Australian Museum. Occurrence record: Malacology:C.150357 Okenia hallucigenia. 1986. Retrieved from http://biocache.ala.org.au/occurrences/3105c430-af0d41c7-9a52-819c9e4c7523 on 10 July 2015.

Australian Museum. Occurrence record: Malacology:C.159212 Marionia pustulosa. 1989. Retrieved from http://biocache.ala.org.au/occurrences/7bdee103-19d04cc9-b9b9-f776d960017f on 10 July 2015.

Australian Museum. Occurrence record: wSPUISAw Facelina rhodopos. 2002. Retrieved from http://biocache.ala.org.au/occurrences/6f067294-1e08-4238a343-ed6762b29136 on 9 July 2015.

Baba K. Opisthobranchia of Japan (II). J Dept Agric Kyushu Imp Univ. 1937;5:289-344

Baba K. Opisthobranchia of Sagami Bay. Iwanami Shoten: Tokyo, Japan; 1949. p. 289-383.

Baba K. Opisthobranchia of Sagami Bay, Supplement. Tokyo: Iwanami Shoten; 1955. p. 59

Baird A, Sommer B, Madin J. Pole-ward range expansion of Acropora spp. along the east coast of Australia. Coral Reefs. 2012;31:1063.

Behrens DW, Petrinos C, Schrurs C. Nudibranch behavior. Jacksonville FL, USA: New World Pubns Inc; 2005. p. 67.

Bidgrain P. 2006. South-West Indian Ocean Seaslugs - Facelina rhodopos. Retrieved from http://seaslugs.free.fr/nudibranche/a_face_rhodopos.htm on 8 July 2015.

Booth D, Figueira W, Gregson M, Brown L, Beretta G. Occurrence of tropical fishes in temperate southeastern Australia: role of the East Australian Current Estuar Coast Shelf Sci. 2007;72:102-14.

Burn R. A checklist and bibliography of the Opisthobranchia (Mollusca: Gastropoda) of Victoria and the Bass Strait area, south-eastern Australia. Museum Vic Sci Reports. 2006;10:7-13.

Camacho-García YE, Gosliner TM. Systematic revision of Jorunna Bergh, 1876 (Nudibranchia: Discodorididae) with a morphological phylogenetic analysis. J Molluscan Stud. 2008:74:143-81.

Camacho-García YE, Ornelas-Gatdula E, Gosliner TM, Valdés A. Phylogeny of the family Aglajidae (Pilsbry, 1895) (Heterobranchia: Cephalaspidea) inferred from mtDNA and nDNA. Mol Phylogenet Evol. 2013;71:113-26.

Chan JM, Gosliner TM. Preliminary phylogeny of Thordisa (Nudibranchia: Discodorididae) with descriptions of five new species. Veliger. 2007:48:284-308.

Cobb G, Mullins D. Nudibranchs: Sunshine Coast QLD \& Tasmania Australia Jorunna ramicola. 2009. Retrieved from http://www.nudibranch.com.au/ pages/6577a.htm on 07 July 2015.

Cobb G, Mullins D. Nudibranchs: Sunshine Coast QLD \& Tasmania Australia Kaloplocamus peludo. 2012a. Retrieved from http://www.nudibranch.com.au/ pages/DSC09615w.htm on 29 July 2015.

Cobb G, Mullins D. Nudibranchs: Sunshine Coast QLD \& Tasmania Australia Marionia pustulosa. 2009. Retrieved from http://www.nudibranch.com.au/ pages1/MARIONI1.htm on 8 July 2015

Cobb G, Mullins D. Nudibranchs: Sunshine Coast QLD \& Tasmania Australia Polycera risbeci. 2009. Retrieved from http://www.nudibranch.com.au/pages1/ MoolLedCap258w.htm on 29 July 2015

Cobb G, Mullins D. Nudibranchs: Sunshine Coast QLD \& Tasmania Australia Tambja victoriae. 2003. Retrieved from http://www.nudibranch.com.au/pages/ 4367.htm on 29 July 2015

Cobb G, Mullins D. Nudibranchs: Sunshine Coast QLD \& Tasmania Australia Thordisa tahala. 2009. Retrieved from http://www.nudibranch.com.au/pages/ CapThorClown-044w.htm on 8 July 2015.

Cobb G, Mullins D. Nudibranchs: Sunshine Coast QLD \& Tasmania Australia Trinchesia puellula. 2008. Retrieved from http://www.nudibranch.com.au/ pages/Capture_00149.htm on 8 July 2015.

Cobb G, MullinsD. Nudibranchs: Sunshine Coast QLD \& Tasmania Australia Sakuraeolis nungunoides. 2012b. Retrieved from http://www.nudibranch.com. au/pages/G_3731c.htm on 29 July 2015.

Coleman N. Nudibranchs encyclopedia. Qld, Australia: Neville Coleman's Underwater Geographic: Springwood; 2008. p. 416.

Coleman N, Marsh N. Diving Australia : a guide to the best diving Down Under. Basingstoke, UK: Periplus Editions; 1997. p. 267.

Creese R, Wales NS. Mapping the habitats of NSW estuaries. Industry \& Investment NSW: Sydney; 2009. p. 1-95.
Davis TR, Harasti D, Smith SDA. Developing a habitat classification typology for subtidal habitats in a temperate estuary in New South Wales. Marine and Freshwater Research: Australia; 2015. http://dx.doi.org/10.1071/ MF15123.

DECCW. Port Stephens - Great Lakes Marine Park Operational Plan. Department of Environment, Climate Change and Water/New South Wales Marine Parks Authority: Sydney South; 2010

Figueira WF, Booth DJ. Increasing ocean temperatures allow tropical fishes to survive overwinter in temperate waters. Glob Chang Biol. 2010;16:506-16.

Gladstone W. Requirements for marine protected areas to conserve the biodiversity of rocky reef fishes. Aquat Conserv Mar Freshwat Ecosyst. 2007; 17(1):71-87.

Good P. Sakuraeolis nungunoides. 2012. Retrieved from http://www. goodpicturesonline.com/Branchs/Nudibranchia 29 July 2015

Good P. Thordisa tahala. 2015. Retrieved from http://www.goodpicturesonline. com/Branchs/Nudibranchia 07 July 2015

Gosliner T. Nudibranchs of southern Africa: a guide to opisthobranch molluscs of southern Africa. California Academy of Sciences, San Francisco, USA: Sea Challengers; 1987. p. 1-136.

Gosliner TM, Vallès Y. Shedding light onto the genera (Mollusca: Nudibranchia) Kaloplocamus and Plocamopherus with description of new species belonging to these unique bioluminescent dorids. Veliger. 2006;48:178-205.

Gosliner TM, Behrens DW, Valdés Á. Indo-Pacific nudibranchs and sea slugs: a field guide to the world's most diverse fauna. California Academy of Sciences, San Francisco, USA: Sea Challengers Natural History Books; 2008. p. 1-425.

Harasti D. Range extension and first occurrence of the thorny seahorse Hippocampus histrix in New South Wales, Australia. Marine Biodiversity Records. 2015:8(E49):3.

Harasti D, Gladstone W. Does underwater flash photography affect the behaviour movement and site persistence of seahorses? J Fish Biol. 2013:83:1344-53.

Harasti D, Martin-Smith K, Gladstone W. Ontogenetic and sex-based differences in habitat preferences and site fidelity of White's seahorse Hippocampus whitei. J Fish Biol. 2014;85(5):1413-28.

Harasti D, Malcolm H, Gallen C, Coleman MA, Jordan A, Knott NA. Appropriate set times to represent patterns of rocky reef fishes using baited video. J Exp Mar Biol Ecol. 2015;463:173-80.

Hobday AJ, Lough JM. Projected climate change in Australian marine and freshwater environments. Mar Freshw Res. 2011:62:1000-14.

MAGNT. Occurrence record: Mollusc:P023592 Thecacera pacifica. 2003. Retrieved from http://biocache.ala.org.au/occurrences/5893262b-8b82-44bb-9f6789c4a6284f3e on 02 August 2015.

MAGNT. Occurrence record: Mollusc:P031745 Trinchesia ornata. 2011. Retrieved from http://biocache.ala.org.au/occurrences/214759ad-1e55-48e2-9d02eca137b108f7 on 8 July 2015

MAGNT. Occurrence record: Mollusc:P054648 Polycera melanosticta. 2014 Retrieved from http://biocache.ala.org.au/occurrences/76b2a97c-3b3e-4d478c39-f578bbd0723f on 05 August 2015.

Malcolm HA, Jordan A, Smith SD. Biogeographical and cross-shelf patterns of reef fish assemblages in a transition zone. Mar Biodivers. 2010;40:181-93.

Marcus E. Opisthobranch mollusks from California. Veliger. 1961;3(Supplement 1):1-85.

Marshall JG, Willan RC. Nudibranchs of Heron Island, Great Barrier Reef: A Survey of the Opisthobranchia (Sea Slugs) of Heron and Wistari Reefs. Backhuys: Leiden, The Netherlands; 1999. p. 1-257.

Miller MC. The dorid nudibranch genus Jorunna Bergh, 1876 (Gastropoda: Opisthobranchia) in New Zealand. J Nat Hist. 1996;30:1095-109.

Morton JK, Gladstone W. Spatial, temporal and ontogenetic variation in the association of fishes (family Labridae) with rocky-reef habitats. Mar Freshw Res. 2011;62(7):870-84

Museum Victoria. Occurrence record: Invertebrates:F29710 Thecacera pacifica. 1975a. Retrieved from http://biocache.ala.org.au/occurrences/1d9881383cb7-4cc9-ac02-45921156ee2f on 02 August 2015.

Museum Victoria. Occurrence record: Invertebrates:F79379 Trinchesia ornata. 1980 Retrieved from http://biocache.ala.org.au/occurrences/fcc53ef6-4e86-4a7e9415-c4a57fb7871c on 8 July 2015

Nimbs MJ, Willan RC, Smith SDA. Range extensions for heterobranch sea slugs (formerly opisthobranch) belonging to the families Diaphanidae, Plakobranchidae and Facelinidae on the eastern coast of Australia. Mar Biodivers Rec. 2015;8, e76.

Nudi Pixel. Aegires incusus. 2009a. Retrieved from http://www.nudipixel.net/ photo/00019707/ 5 August 2015. 
Nudi Pixel. Ceratosoma tenue. 2008a. Retrieved from http://www.nudipixel.net/ photo/00004051/ 5 August 2015.

Nudi Pixel. Eubranchus ocellatus. 2010a. Retrieved from http://www.nudipixel.net/ photo/00025974/ 5 August 2015.

Nudi Pixel. Facelina rhodopos. 2011a. Retrieved from http://www.nudipixel.net/ species/facelina_rhodopos/ 08 July 2015.

Nudi Pixel. Noumea laboutei. 2008c. Retrieved from http://www.nudipixel.net/ photo/00004126/location/nelson_bay/ 5 August 2015.

Nudi Pixel. Philiniopsis reticulata. 2004. Retrieved from http://www.nudipixel.net/ photo/00004145/location/nelson_bay/ 05 August 2015.

Nudi Pixel. Platydoris ellioti. 2010b. Retrieved from http://www.nudipixel.net/ photo/00028026/location/nelson_bay/on 5 August 2015.

Nudi Pixel. Pleurobranchus forskalii. 2012. Retrieved from http://www.nudipixel. net/photo/00040836/location/nelson_bay/ 5 August 2015.

Nudi Pixel. Risbecia tryoni. 2010c. Retrieved from http://www.nudipixel.net/photo/ 00026294/location/nelson_bay/ 5 August 2015.

Nudi Pixel. Tambja victoriae. 2003. Retrieved from http://www.nudipixel.net/ photo/00028824/ 30 July 2015.

Nudi Pixel. Tambja victoriae. 2008d. Retrieved from http://www.nudipixel.net/ photo/00009581/ 30 July 2015.

Nudi Pixel. Thecacera pacifica. 2010d. Retrieved from http://www.nudipixel.net/ photo/00026516/ 02 August 2015.

Nudi Pixel. Thecacera pacifica. 2011c. Retrieved from http://www.nudipixel.net/ photo/00033497/ 02 August 2015.

Nudi Pixel. Trinchesia ornata. 2011d. Retrieved from http://www.nudipixel.net/ species/trinchesia_ornata/ 08 July 2015.

Nudi Pixel. Trinchesia yamasui. 2009b. Retrieved from http://www.nudipixel.net/ photo/00037841/location/nelson_bay/ 5 August 2015.

Odhner NH. Nudibranchia Dendronotacea. A revision of the system. Memoires du Musee Royal d'Histoire Naturelle de Belgique, series 2, fasc. 3. 1936. p. 1057-1128

O'Hara T. Marine invertebrate conservation at San Remo. Vic Nat. 1995;112:50-3.

Pitt NR, Poloczanska ES, Hobday AJ. Climate-driven range changes in Tasmanian intertidal fauna. Mar Freshw Res. 2010;61:963-70.

Pola M, Cervera JL, Gosliner TM. Four new species of Tambja Burn, 1962 (Nudibranchia: Polyceridae) from the Indo-Pacific. J Molluscan Stud. 2005;71:257-67.

Pola M, Roldan P, Padilla S. Molecular data on the genus Okenia (Nudibranchia: Goniodorididae) reveal a new cryptic species from New South Wales (Australia). J Mar Biol Assoc U K. 2014;94(3):587-98.

Poulos D, Gallen C, Davis T, Booth D, Harasti D. Distribution and spatial modelling of a soft coral habitat in the Port Stephens-Great Lakes Marine Park: implications for management. Mar Freshwater Res. 2015. doi.org/10.1071/ MF14059.

Przeslawski R, Ahyong S, Byrne M, Woerheide G, Hutchings P. Beyond corals and fish: the effects of climate change on noncoral benthic invertebrates of tropical reefs. Glob Chang Biol. 2008;14:2773-95.

Riek D. Discodorididae: Jorunna ramicola Miller, 1996. 2013a. Retrieved from http://www.roboastra.com/Opisthobranch4/hpop2104.htm on 07 July 2015.

Riek D. Discodorididae: Thordisa tahala Chan \& Gosliner, 2006. 2013b. Retrieved from http://www.roboastra.com/Opisthobranch4/brop275.html on 07 July 2015.

Riek D. Facelinidae, Sakuraeolis nungunoides. 2013d. Retrieved from http://www. roboastra.com/Opisthobranch6/brop311.html on 29 July 2015.

Riek D. Facelinidae, Facelina rhodopos Yonow, 2000. 2013c. Retrieved from http:// www.roboastra.com/Opisthobranch6/hpop461.html on 09 July 2015.

Riek, D. Polyceridae, Polycera risbeci Odhner, 1941. 2013e. Retrieved from http:// www.roboastra.com/Opisthobranch3/hpop914.html on 09 July 2015.

Rudman WB. 2005. Tambja victoriae Pola, Cervera \& Gosliner, 2005. Retrieved from http://www.seaslugforum.net/find/tambvict on 30 July 2015.

Rudman WB. Aglaja? orientalis from Japan. 2003f. 2003f. Retrieved from http:// www.seaslugforum.net/find/8843 on 05 August 2015.

Rudman WB. Cerberilla ambonensis from New South Wales. 2010. Retrieved from http://www.seaslugforum.net/find/23379 on 05 August 2015.

Rudman WB. Chromodoris at Fly Point. 1998a. Retrieved from http://www. seaslugforum.net/find/37 on 05 August 2015.

Rudman WB. Chromodoris verrieri from Nelson Bay. 2003a. Retrieved from http:// www.seaslugforum.net/find/9244 on 05 August 2015.

Rudman WB. Colour form of Chromodoris striatella. 2000a. Retrieved from http:// www.seaslugforum.net/find/2386 on 05 August 2015.

Rudman WB. Crosslandia? from Port Stephens. 1999a. Retrieved from http://www. seaslugforum.net/find/775 on 05 August 2015.
Rudman WB. Cuthona puellula (Baba, 1955). 2002d. Retrieved from http://www. seaslugforum.net/showall/cuthpuel on 03 August 2015.

Rudman WB. Cuthona sibogae (Bergh, 1905). 2003b. Retrieved from http://www. seaslugforum.net/showall/cuthsp4 on 05 August 2015.

Rudman WB. Dermatobranchus nigropunctatus Baba, 1949. 2004a. Retrieved from http://www.seaslugforum.net/find/dermnigr on 05 August 2015.

Rudman WB. Discodoris from Nelson Bay. 1998b. Retrieved from http://www. seaslugforum.net/message/51 on 05 August 2015.

Rudman WB. Facelina rhodopos from Malaysia. 2002a. Retrieved from http://www. seaslugforum.net/find/8914 on 09 July 2015.

Rudman WB. Feeding habits of Nembrotha rosannulata. 2008. Retrieved from http://www.seaslugforum.net/find/12735 on 05 August 2015.

Rudman WB. Kaloplocamus peludo from Heron Island. 2007. Retrieved from http://www.seaslugforum.net/find/19552 on 29 July 2015.

Rudman WB. Nembrotha rutilans mating. 1999b. Retrieved from http://www. seaslugforum.net/find/774 on 05 August 2015.

Rudman WB. Notobryon wardi Odhner, 1936. 1999c. Retrieved from http://www. seaslugforum.net/find/notoward on 05 August 2015.

Rudman WB. Noumea varians from New South Wales. 1999d. Retrieved from http://www.seaslugforum.net/find/1682 on 05 August 2015.

Rudman WB. Okenia purpurata - new record. 2006b. Retrieved from http://www. seaslugforum.net/find/15897 on 05 August 2015.

Rudman WB. Okenia vena from Port Stephens, NSW. 2006c. Retrieved from http:// www.seaslugforum.net/find/15907 on 05 August 2015.

Rudman WB. Pikachu was inspired by this? 2003c. Retrieved from http://www. seaslugforum.net/find/10903 on 30 July 2015.

Rudman WB. Polybranchia orientalis from eastern Australia. 1999e. Retrieved from http://www.seaslugforum.net/find/1003 on 05 August 2015.

Rudman WB. Re: Striped and unstriped Lobiger viridis together. 2009. Retrieved from http://www.seaslugforum.net/find/22944 on 05 August 2015.

Rudman WB. Sakuraeolis nungunoides Rudman, 1980. 2003d. Retrieved from http://www.seaslugforum.net/find/sakunung on 09 July 2015.

Rudman WB. Thecacera pacifica Bergh, 1883. 2002c. Retrieved from http://www. seaslugforum.net/find/thecpaci on 07 July 2015.

Rudman WB. Thorunna florens from Port Stephens, NSW. 2003e. Retrieved from http://www.seaslugforum.net/find/9873 on 05 August 2015.

Rudman W, Willan R. Opisthobranchia. In: Mollusca: The southern synthesis. Melbourne, Australia: Fauna of Australia. CSIRO; 1998. p. 915-1035.

Scott A, Harasti D, Davis T, Smith SDA. Southernmost records of the host sea anemone, Stichodactyla haddoni, and associated commensal shrimps in a climate change hotspot. Mar Biodivers. 2015;45:145-6.

Simonitch S. Sea Slug Bears Striking Resemblance To Pikachu, Rightfully Known As 'Pikachu Sea Slug' in Japan. Rocket News 24 ピカチユウが 「ウデフリッノザヤウミウシ」に激似と話題: Tokyo. 2012. Retrieved from http://en.rocketnews24.com/2012/01/24/sea-slug-bears-strikingresemblance-to-pikachu-rightfully-known-as-pikachu-sea-slug-in-japan/ 5 August 2015.

Smith SDA. Rapid assessment of invertebrate biodiversity on rocky shores: where there's a whelk there's a way. Biodivers Conserv. 2005;14(14):3565-76.

Smith SDA. Interpreting molluscan death assemblages on rocky shores: are they representative of the regional fauna? J Exp Mar Biol Ecol. 2008a;366(1):151-9.

Smith S. Live ovulids from Nelson Bay, NSW. Newsletter Malacol Soc Aust. 2008b;134:1.

Smith SDA, Edgar BE. Documenting the density of subtidal marine debris across multiple marine and coastal habitats. PLoS One. 2014;9, e94593.

Smith SDA, Jordan A, Creese RG, Gladstone W. The Marine Environment of the Hunter-Central Rivers Region of New South Wales: A Review of Current Knowledge. Report to the: Hunter-Central Rivers Catchment Management Authority; 2010. p. 190. ISBN 978-1-921324-02-4.

Thompson T. Eastern Australian Dendronotoidea (Gastropoda: Opisthobranchia). Zool J Linnean Soc. 1972;51:63-77.

Underwood AJ, Chapman M. Intertidal Temperate Rocky Shores. In: Marine Ecology. Melbourne, Australia: Oxford University Press; 2007. p. 402-27.

Vila-Concejo A, Short AD, Hughes MG, Ranasinghe R. Shoreline implications of flood-tide delta morphodynamics. The case of Port Stephens (SE Australia). Coastal Sediments. 2007;1417-1430.

Wells FE, Bryce CW. Sea slugs of Western Australia: a guide for species from the Indian to West Pacific Oceans. Perth, W.A: Western Australian Museum; 2000. p. 1-184. 
Willan RC. The opisthobranch Thecacera pennigera (Montagu) in New Zealand, with a discussion on the genus. Veliger. 1976;18(4):347-52.

Willan RC, Dollimore JM, Nicholson J. A survey of fish populations at Karikari Peninsula, Northland, by scuba diving. N Z J Mar Freshw Res. 1979;13(3): 447-56.

Yonow N. Red Sea Opisthobranchia 4: the orders Cephalaspidea, Anaspidea, Notaspidea and Nudibranchia: Dendronotacea and Aeolidacea. Fauna Arabia. 2000;18:87-132

Submit your next manuscript to BioMed Central and we will help you at every step:

- We accept pre-submission inquiries

- Our selector tool helps you to find the most relevant journal

- We provide round the clock customer support

- Convenient online submission

- Thorough peer review

- Inclusion in PubMed and all major indexing services

- Maximum visibility for your research

Submit your manuscript at www.biomedcentral.com/submit 